\title{
Chromovitrectomy in Vitreous Loss During Cataract Surgery
}

\author{
Taiji Sakamoto ${ }^{1}$ and Toshio Hisatomi ${ }^{2}$ \\ ${ }^{1}$ Department of Ophthalmology, \\ Kagoshima University Graduate School of Medical and Dental Sciences, Kagoshima, \\ ${ }^{2}$ Department of Ophthalmology, \\ Kyushu University Graduate School of Medicine, Fukuoka, \\ Japan
}

\section{Introduction}

Cataract surgery is the commonest surgical operation performed throughout the world, but surgery has always involved some risk. For example, vitreous loss (vitreous breaching the posterior capsule) associated with capsular rupture is a complication that significantly affects postoperative visual acuity. Direct and immediate effects of vitreous loss involve deviation of the pupil (corectopia), inflammation of the anterior chamber, corneal damage, and intraocular pressure elevation; indirect and late effects are cystoid macular edema, vitreous inflammation, and retinal detachment [1-6].

Various factors have been reported to associated with this complication [1]. There is no doubt that the skill of the surgeon is a major factor, and other predisposing factors include white cataract, small pupil, pseudoexfoliation, and a history of previous intraocular surgery. When lost vitreous is treated improperly, it can cause secondary complications, resulting in poor final visual acuity. To prevent these secondary harmful results, good knowledge, experience, and skill are required from surgeons. Specifically, complete removal of the vitreous from the anterior chamber is essential for a favourable postoperative outcome after posterior capsule rupture. However, the surgical procedure for removing the vitreous is difficult because the structure appears transparent under an operating microscope. The vitreous body in the anterior chamber is sometimes difficult even for an experienced surgeon to identify because the cornea is often damaged incidentally in cases involving vitreous prolapse, which are by definition complex cases.

To facilitate this procedure, several approaches have been applied to improve visualisation of lost vitreous. A vital dye has generally been used to stain the lens capsule in standard cataract surgery. This technique is adopted to visualize lost vitreous. Another approach is to stain the components of the vitreous body directly. Recently, triamcinolone acetonide (TA) has been used as an adjunct to visualize the vitreous body during pars plana vitrectomy (PPV) [7]. This technique was originally developed to visualize the vitreous body on the retinal surface. Moreover, it was found to be very helpful for elucidating the pathophysiology of the vitreous body in various retinal diseases [8-10]. Therefore, it has been a natural step to adopt this technique to stain the vitreous in an attempt to facilitate its 
removal in cases of a ruptured capsule during cataract surgery. The following section presents the concepts behind this methods as well as technical details.

\section{History of visualization of the vitreous body}

Modern closed vitreous surgery owes much to the work of Machemer [11]. After initial success vitrectomy continued to be developed and has now been established as a standard treatment for vitreoretinal diseases. With advances in instrumentation and understanding of vitreoretinal pathophysiology the indications for vitrectomy have been expanded to include a variety of diseases such as simple rhegmatogenous retinal detachment, macular hole, and macular oedema [8]. In the early days of its use vitrectomy was performed for diseases in which the vitreous body was visible due to vitreous hemorrhage, proliferative diabetic retinopathy, and asteroid hyalosis. At present though indications for vitrectomy include eyes with optically clear vitreous such as in macular hole surgery, and removing transparent hyaloid and/or internal limiting membrane (ILM) has become a standard procedure. Despite advances in surgical instrumentation and techniques, the vitrectomy procedure continues to demand both skill and experience. Removing the vitreous is a particularly challenging procedure, partly due to its transparency. Various approaches have been taken to assist in visualisation of the vitreous during vitrectomy. Recently, intraoperative triamcinolone acetonide has been used in many countries worldwide $[7,8]$.

In the earliest years of retinal surgery, it was recognized that vital dyes could be helpful for facilitating surgery. In such cases, the structure of the vitreous was not the main target, so dyes such as Indian ink, methylene blue and fluorescein were used primarily to visualise retinal breaks. This vital staining was not so commonly used in surgery at that time; however, recently it has been revived as chromovitrectomy. Visualizing ILM is being studied extensively and chromovitrectomy is now one of the most important elements of vitreous surgery.

In terms of the macroscopic structure of the vitreous, important work has been done using cadaveric eyes and living eyes with biomicroscopy or optical coherence tomography (OCT) examinations; the results have contributed extensively to our understanding of the pathology of vitreoretinal diseases [12-14]. Unfortunately, observational techniques cannot be applied during surgery, thus valuable information cannot be fully used during surgery in individual cases, especially during surgical manoeuvres, though of note intraoperative OCT is starting to be used by some surgeons. Abrams et al noticed in the early days of vitrectomy that staining of the vitreous with fluorescein dye improved the ease of vitrectomy by facilitating clear visualization of the vitreous, although this method was used only in animals and for surgical training [15]. Some 20 years later, it was proven in a report which showed that preoperative fluorescein angiography or orally administered sodium fluorescein stained a transparent vitreous, making it visible as light green [16,17]. However, thin posterior hyaloids could not be visualized well by this method, which is indispensable in modern surgery on the retinal surface such as for macular hole or pre-retinal membrane. On the other hand, intravitreous application of autologous blood was used for a similar purpose [18]. It was reported that intravitreous autologous blood could identify the posterior vitreous cortex and facilitate surgical manoeuvres with reduced complications. However, blood is not transparent so it is not necessarily helpful to remove ILM. Furthermore, unclotted erythrocytes may become lysed in the irrigation fluid and swirl the liberated haemoglobin in the vitreous cavity, obscuring the fundus view with a dusty 
appearance. This characteristic prevents autologous blood from being commonly used in vitrectomy.

\section{Visualization of vitreous during cataract surgery}

Although vitreous loss is a significant complication of cataract surgery, the outcome can be almost as good as if it had not happened when managed properly - though there nevertheless remains an increased risk of retinal break and retinal detachment. Needless to say, the first and most important step is early recognition that vitreous loss has taken place. The second step is to evaluate the loss of vitreous properly with or without using an anterior vitrector. For that purpose, at present there are the several approaches as described below.

\subsection{Trypan blue}

Trypan blue has been reported to be effective for staining tissue during anterior and posterior segment surgery without significant toxicity to corneal endothelial cells with short exposure times [19]. In a preliminary study, the dye was injected into the anterior chamber under an air bubble, which prevents dilution of the trypan blue and avoids direct contact between the undiluted dye and the endothelium. Subsequently, the dye was removed with a vitreous cutter as vitrectomy proceeded. On the first postoperative day no residual staining was visualized on inspection with a slit lamp and ophthalmoscope, and no side effects related to intraoperative use of the dye were observed in any patient. This technique was applied to visualize prolapsed vitreous in cataract surgery [20]. Trypan blue does not stain vitreous body directly, but rather allows visualization of the vitreous body by staining the surrounding tissues such as iris and lens capsule. So far there have been no reports on the apparent toxicity of trypan blue in intracameral use [21].

\subsection{Triamcinolone acetonide and other related materials}

In Japan triamcinolone acetonide (TA) is now the most commonly used agent for pars plana vitrectomy (PPV) for visualization of a transparent vitreous body. A national survey in Japan showed that 75\% of PPV comprised TA-assisted PPV in 2005 [22]. When intravitreous TA was first used for PPV, there were strong concerns about its potential adverse effects such as glaucoma, endophthalmitis, or direct toxicity related to preservatives [8]. However, the incidence of these potential adverse events were not significant, probably as most of the TA crystals were removed at the end of surgery [8]. This safety issue was partly supported by the fact that intravitreous injection of TA (without removal) did not cause serious or profound adverse events [23].

Unlike other vital dyes, white granules of TA adhere to the surface of the vitreous, which clearly shows the margin of the vitreous $[24,25]$ This is also the case with intracameral use of TA. Even a small amount of TA can be of great help in visualizing even traces of vitreous in the anterior chamber with a surgical microscope (Figure 1). Theoretically, TA crystals should be removed as much as possible at the end of surgery, because steroid granules left in the anterior chamber might induce secondary glaucoma or infection. But, it might not be necessary because an intracameral depot to release dexamethasone is left safely in the anterior chamber after cataract surgery [26]. In addition, it is impossible in practice to remove all the granules from the anterior chamber. 

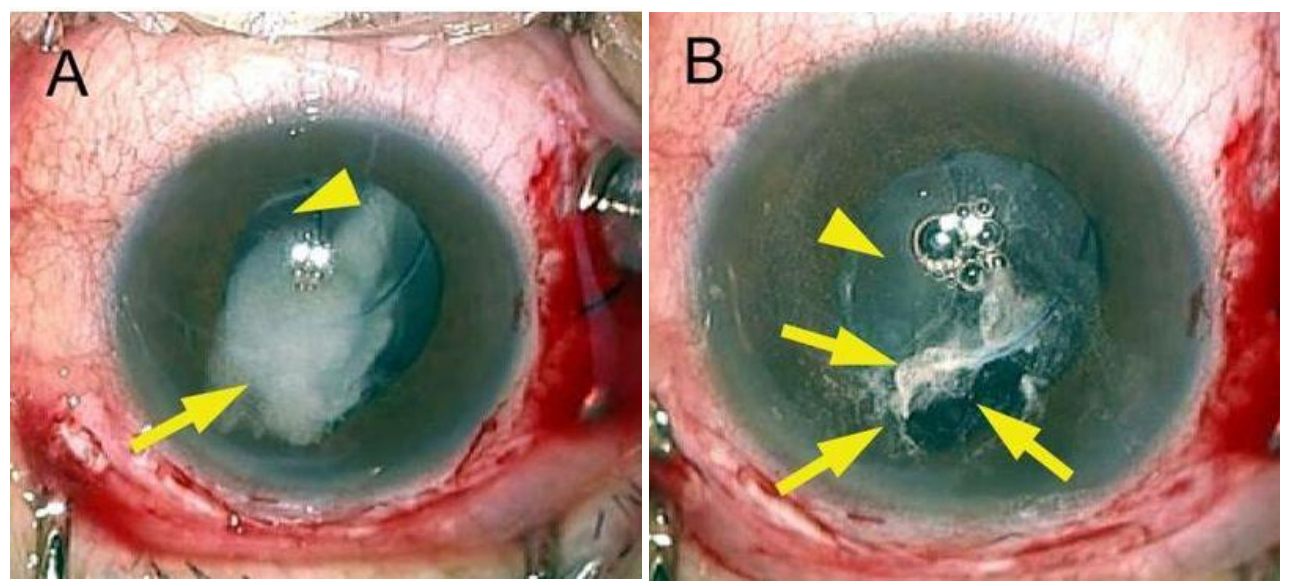

The posterior capsule is ruptured and the vitreous body is moved into the anterior chamber; triamcinolone is sprayed at the location where the vitreous body is likely to have been placed, using a syringe with a dull-edged needle. The vitreous body immediately appears as a white-coloured gel. The vitreous body with TA crystals (arrows) were then easily removed with a vitreous cutter. Arrowhead indicates optic of intraocular lens (A and B). Reprinted from: Yamakiri K et al. (2004), with permission from Elsevier [25].

Fig. 1. Intraoperative photograph of triamcinolone-assisted vitrectomy (cataract surgery after rupture of lens capsule).

A novel approach related to use of TA will be described. Kaji et al reported a new technique for visualising the vitreous in the anterior chamber using 11-deoxycortisol (11-DC) [27]. Its suspension can be prepared without the antiseptic and emulsifying agents contained in commercially available TA. A precursor of cortisol in the cascade of steroid metabolism, 11DC, is naturally detectable in blood and urine. This material is free from glucocorticoid and mineralocorticoid actions. In addition, the conversion system of 11-DC into cortisol is not present in ocular tissues. Thus, 11-DC injected into the anterior chamber does not induce steroid-related complications such as ocular hypertension. As for the molecular structure, 11-DC contains hydroxyl groups, thus it has some hydrophilic characteristics. Therefore, 11DC left in the anterior chamber is easily cleared and excreted through the urine. In their study, there were white particles on the surface of the iris or in the vitreous body on the day following surgery, but all the particles had completely disappeared within 2 days after surgery [27]. This hydrophilic characteristic of 11-DC is highly advantageous over hydrophobic cholesterol as a tool to visualize the vitreous body. 
There are however concerns about use of 11-DC. Under some circumstances, the steroid activity of TA is advantageous to reduce post-operative inflammation, which 11-DC does not have. The particle size of 11-DC is larger than that of TA. Above all, 11-DC is not commercially available in most of countries at present. Although the use of TA in cataract surgery was off-label, there is a product of TA for general medical use (e.g. Kenalog made by Bristol-Myers Squibb) and products which are licensed specifically for intraocular conditions have also been licensed (e.g. Trivaris made by Allergan). Consequently, TA can be used more easily and in theory more safely than 11-DC. This is a major obstacle to more widespread use of 11-DC.

In an experimental study, Yamashita et al demonstrated that polylactic acid could be applied for this purpose [28]. Polylactic acid is a biodegradable substance without any biological effects. In 2004, the U.S. Food and Drug Administration approved a poly-L-lactic acid (PLLA)-based injectable medical device for restoring and/or correcting signs of facial fat loss in people with human immunodeficiency virus. As a result, the properties of PLLA microparticles have attracted considerable interest in the medical community. PLLA granules are originally white and adhere to the gel-like structure of the vitreous body so easily that they assist in visualizing the transparent vitreous as do TA granules but with no harmful consequences in rabbits and nonhuman primates. In future this agent might be a good alternative in cases where TA is proven to be intolerably harmful such as ocular steroid-responders at risk of glaucoma.

\section{Possible adverse events}

Although chromovitrectomy is very helpful for facilitating the removal of vitreous in the anterior chamber, to our knowledge there has been no large-scale comparative study. The incidence of vitreous loss in cataract surgery is low and this complication is not predictable [29], therefore it is not possible to conclusively describe any role for chromovitrectomy in preventing adverse events. However, possible adverse events can be described from the results of animal studies, small studies, or chromovitrectomy for vitreoretinal diseases.

\subsection{Trypan blue and other vital dyes}

At present indocyanine green, trypan blue, and brilliant blue $G$ are commonly used for capsular staining in cataract surgery. Of these, trypan blue has been deemed safe for clinical use [30]. However, non-infectious endophthalmitis has been reported after intracameral use of trypan blue in cataract surgery [31]. In an in vitro study, trypan blue has been shown to be toxic to human corneal endothelial cells at high concentrations or with long exposure [32]. In an animal study conducted by the authors, intracameral injection of each dye resulted in corneal damage to a certain extent and trypan blue was most toxic (Figure 2) [33]. The results of in vitro or animal studies may not necessarily reflect clinical conditions correctly; however, this information should be noted when using trypan blue. Therefore, trypan blue should be removed as much as possible at the end of surgery. The use of trypan blue in cataract surgery does not increase intraocular pressure, nor cause infectious endophthalmitis, or retinal detachment [34].

Most importantly, it should be remembered that these dyes are used in potentially inflamed and/or damaged tissues. The safety issue should be considered more seriously than usual and great caution is warranted. 


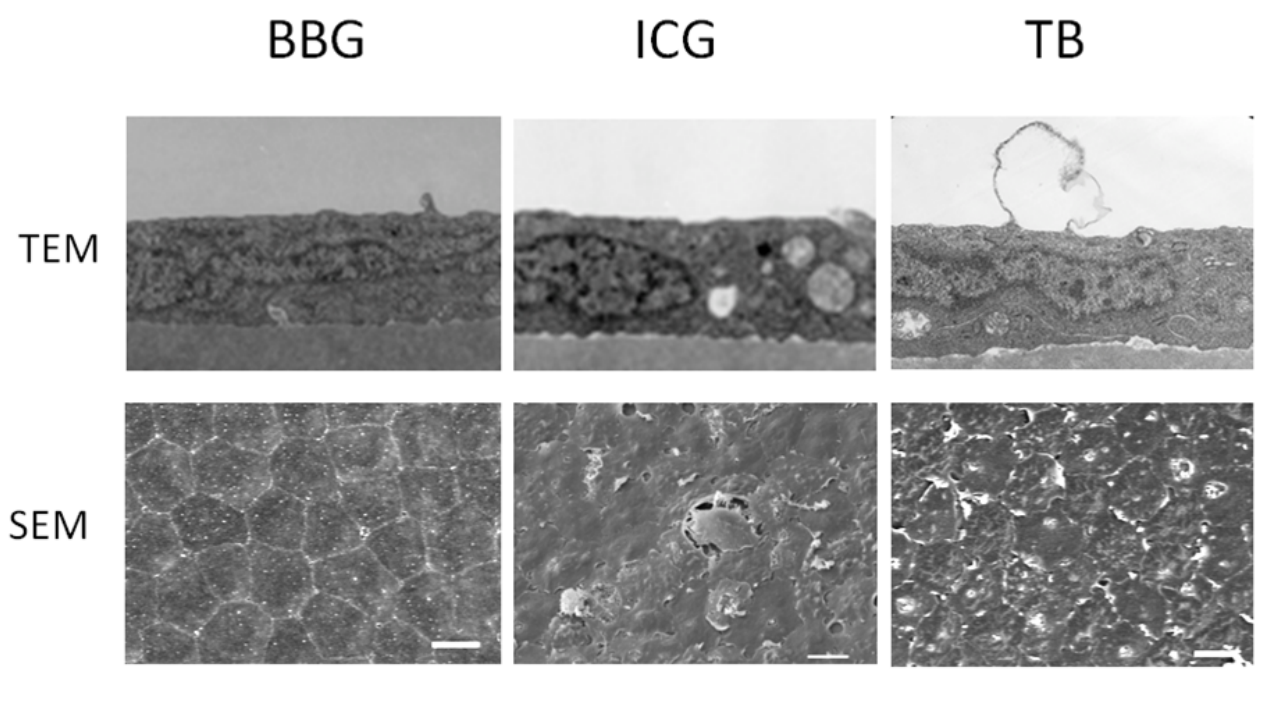

Brown Norway rats received intracameral injection into each eye and the eyes were examined with electron microscopy after 2 months. In the brilliant blue G (BBG) groups, the ultrastructure of the corneal cells and collagen cellular matrix were well preserved $(10 \mathrm{mg} / \mathrm{mL})$. The indocyanine green (ICG) group showed mitochondrial swelling at the $5-\mathrm{mg} / \mathrm{mL}$ concentration. The trypan blue (TB) group showed cyst formation in the endothelial cell layer due to separation of the cells and degeneration of the corneal endothelium in a sporadic manner (original magnification x6600). The BBG-exposed corneas demonstrated a normal hexagonal endothelial cell sheet with intact borders and no endothelial swelling. The ICG group showed cellular swelling and some degenerated cells in the corneal endothelial cells. C, In the TB group, endothelial cell shrinkage was recognized in a sporadic manner. Cell shrinkage led to endothelial cell loss in the central area of the cornea (original magnification x1000). The detailed method was described by Hisatomi et al [33].

Fig. 2. Ultrastructure of rat corneal cells studied using transmission electron microscopy and scanning electron microscopy.

\subsection{Triamcinolone and related materials}

Corticosteroids are known to have several adverse effects on an eye, thus this issue was of particular concern from the beginning. However, most of the results have been obtained from the intravitreous use of TA. These should be interpreted carefully.

Corticosteroids are strong inducers of intraocular pressure (IOP) rise. Recent studies have revealed that intravitreous TA without vitrectomy causes a significant rise of IOP in more than $50 \%$ of eyes [23]. In comparison, the incidence of IOP rise was not high after TAassisted vitrectomy, probably due to the fact that TA crystals were almost totally removed at the end of surgery. The largest-scale controlled trial showed that 23 out of 391 eyes (5.9\%) 
had a significant IOP rise in TA-assisted vitrectomy; while 13 out of 383 eyes (3.4\%) did so in conventional vitrectomy, a difference which did not reach statistical significance [35]. Most of these rises in intraocular pressure could be managed with topical treatment, but some of them required filtration surgery $[8,35]$. It should be noted that patients who had a history of glaucoma were not included in these studies. Because a history of glaucoma is a risk factor for an intraocular hypertensive response to corticosteroid, care must be taken when using TA in patients with such a history.

Corticosteroid has an immune-suppressive effect and endopthalmitis has a poor visual prognosis, thus postoperative infection has been the foremost concern in the intraoperative use of TA. The incidence of acute endophthalmitis was reported to be $7 / 26,819$ cases $(0.026 \%)$ in the Japan national survey [22]. Therefore, it is not as high as that of simple intravitreous TA injection $(0.87 \%)$ described by Moshfeghi et al and is similar to the incidence of 20 gauge vitrectomy without TA (0.018 to $0.05 \%)$ [36].

The toxicity of TA suspension has been reported in an experimental study. Direct exposure to preserved TA in rabbits damaged the corneal endothelial cells probably due to the preservative benzyl alcohol [37,38]. To avoid potential toxicities, it is preferable to use preservative-free TA. In most cases, decantation of preservative prior to injection is considered to be sufficient to avoid potential toxicity. At present, if necessary, benzyl alcohol-free TA is commercially available (Triesence ${ }^{\mathrm{TM}}$, Alcon, Ft Worth, Tx, USA) or (MacuaidTM, Wakamoto, Tokyo, Japan).

\section{Chemovitrectomy in the future: To see is to believe!}

Although cataract surgery is one of the safest procedures in ocular surgery, there is always some risk attached. No surgery can be free from risk, thus every effort has to be made to reduce risk in surgery. Vitreous loss is a significant risk of cataract surgery, but proper treatment can prevent further complications ensuing from loss of vitreous. Visualization of vitreous is the key challenge to surgeons trying to provide optimal technical outcomes in such cases, which can be greatly facilitated by chromovitrectomy. Although the present vital dyes or TA may be replaced by better agents in the future, the concept of chromovitrectomy, "visualizing vitreous," will remain as long as vitrectomy is performed.

\section{Acknowledgement}

Supported in part by a grant from the Research Committee on Chorioretinal Degeneration and Optic Atrophy, Ministry of Health, Labor, and Welfare, Tokyo, Japan; and by a Grantin-Aid for Scientific Research from the Ministry of Education, Science, and Culture of the Japanese Government, Tokyo, Japan.

\section{References}

[1] Maerza AA, Ramanathan A, Bidgood P, Horgan S (2009) Visual outcome in cataract surgery complicated by vitreous loss in a district general hospital. Int Ophthalmol. 29:157-160.

[2] Desai P, Minassian D, Reidy A (1999) National cataract surgery survey 1997-8: a report of the results of the clinical outcomes. Br. J. Ophthalmol. 82:1336-1340. 
[3] Ionides A, Minassian D, Tuft S (2002) Visual outcome following posterior capsule rupture during cataract surgery. Br. J. Ophthalmol. 85: 222-224.

[4] Ang GA, Whyte IF (2006) Effect and outcomes of posterior capsule rupture in a district general hospital setting. J. Cataract Refract. Surg. 32: 623-627.

[5] Tan JH, Karwatowski WS (2002) Phacoemulsification cataract surgery and unplanned anterior vitrectomy - is it bad news? Eye.16: 117-120.

[6] Chan FM, Mathur R, Ku JJ, Chen C et al (2003) Short-term outcomes in eyes with posterior capsule rupture during cataract surgery. J. Cataract Refract. Surg. 29: 537-541.

[7] Sakamoto T, Miyazaki M, Hisatomi T, Nakamura T, Ueno A, et al (2002) Triamcinolone-assisted pars plana vitrectomy improves the surgical procedures and decreases the postoperative blood-ocular barrier breakdown. Graefes Arch. Clin. Exp. Ophthalmol. 240:4234-4239.

[8] Sakamoto T, Ishibashi T (2009) Visualizing vitreous in vitrectomy by triamcinolone. Graefes Arch.. Clin. Exp. Ophthalmol. 247:1153-1163.

[9] Enaida H, Hata Y, Ueno A, Ishibashi T, Torii H, et al (2004) Visualization of the Cloquet canal during triamcinolone-assisted vitrectomy. Arch. Ophthalmol. 122:1564-1565.

[10] Sonoda KH, Sakamoto T, Enaida H, Miyazaki M, Noda Y, et al (2004) Residual vitreous cortex after surgical posterior vitreous separation visualized by intravitreous triamcinolone acetonide. Ophthalmology. 111:226-230.

[11] Michaels RG (1990) History of retinal detachment surgery. In Retinal detachment, Michaels RG, Wilkinson CP, Rice TA (eds) The CV Mosby Company, St. Louis, pp243-323

[12] Eisner G. Biomicroscopy of the peripheral fundus. NewYork, Springer 1973.

[13] Sebag J, Balazs EA (1989) Morphology and ultrastructure of human vitreous fibers. Invest. Ophthalmol. Vis. Sci. 30:1867-1871.

[14] Takahashi M, Jalkh A, Hoskins J, Trempe CL, Schepens CL (1981) Biomicroscopic evaluation and photography of liquefied vitreous in some vitreoretinal disorders. Arch. Ophthalmol. 99:1555-1559.

[15] Abrams GW, Topping T, Machemer R (1978) An improved method for practice vitrectomy. Arch. Ophthalmol. 96:521-525.

[16] Rodrigues EB, Penha FM, Furlani B, Meyer CH, Maia M, Farah ME (2008) Historical aspects and evolution of the application of vital dyes in vitreoretinal surgery and chromovitrectomy. Dev. Ophthalmol. 42:29-34.

[17] Yao Y, Wang ZJ, Wei SH, Huang YF, Zhang MN (2007) Oral sodium fluorescein to improve visualization of clear vitreous during vitrectomy for proliferative diabetic retinopathy. Clin. Exp. Ophthalmol. 35:824-827.

[18] Ryan EA, Lee S, Chern S (1995) Use of intravitreal autologous blood to identify posterior cortical vitreous in macular hole surgery. Arch. Ophthalmol. 113:822-823.

[19] Melles GR, de Waard PW, Pameyer JH, Houdijn Beekhuis W (1999) Trypan blue capsule staining to visualize the capsulorhexis in cataract surgery. J. Cataract Refract. Surg. 25:7-9. 
[20] Cacciatori M, Chadha V, Bennett HG, Singh J (2006) Trypan blue to aid visualization of the vitreous during anterior segment surgery. J. Cataract Refract. Surg. 32:389-391.

[21] Norn MS (1980) Per operative trypan blue vital staining of corneal endothelium. Eight years' follow up. Acta. Ophthalmol. (Copenh) 58:550-555.

[22] Sakamoto T, Hida T, Tano Y, Negi A, Takeuchi S, Ishibashi T, et al (2007) Committee for Triamcinolone Acetonide for Ocular Disease in Japan. Survey of triamcinolone acetonide for ocular diseases in Japan. Nippon Ganka Gakkai Zasshi. 111:936-945.

[23] Jonas JB, Kreissig I, Degenring R (2005) Intravitreal triamcinolone acetonide for treatment of intraocular proliferative, exudative, and neovascular diseases. Prog. Retina. Eye Res. 24:587-611.

[24] Burk SE, Da Mata AP, Snyder ME, Schneider S, Osher RH, et al (2003) Visualizing vitreous using Kenalog suspension. J. Cataract Refract. Surg. 29:645-651.

[25] Yamakiri K, Uchino E, Kimura K, Sakamoto T (2004) Intracameral triamcinolone helps to visualize and remove the vitreous body in anterior chamber in cataract surgery. Am. J. Ophthalmol. 138:650-652.

[26] Wadood AC, Armbrecht AM, Aspinall PA, Dhillon B (2004) Safety and efficacy of a dexamethasone anterior segment drug delivery system in patients after phacoemulsification. J. Cataract Refract. Surg. 30:761-768.

[27] Kaji Y, Hiraoka T, Okamoto F, Sato M, Oshika T (2005) Clinical application of 11deoxycortisol in visualizing prolapsed vitreous body after posterior capsule rupture in cataract surgery. J. Cataract Refract. Surg. 31:1133-1138.

[28] Yamashita T, Sakamoto T, Yamakiri K, Miura M, Enaida H, et al (2007) Polylactic acid for visualizing the vitreous body during vitrectomy. Invest. Ophthalmol. Vis. Sci. 48:327732-82.

[29] Jacobs PM (2008) Vitreous loss during cataract surgery: prevention and optimal management. Eye 22: 1286-1289.

[30] Ozturk F, Osher RH (2006) Capsular staining: recent developments. Curr. Opin. Ophthalmol. 17:42-44.

[31] Wu L, Velasques R, Montoya O (2008) Non-infectious endophthalmitis associated with trypan blue use in cataract surgery. Int. Ophthalmol. 28: 89-93.

[32] van Dooren BT, Beekhuis WH, Pels E (2004) Biocompatibility of trypan blue with human corneal cells. Arch. Ophthalmol.122:736-742.

[33] Hisatomi T, Enaida H, Matsumoto H, Kagimoto T, Ueno A, et al (2006) Staining ability and biocompatibility of brilliant blue G: preclinical study of brilliant blue $\mathrm{G}$ as an adjunct for capsular staining. Arch. Ophthalmol.124:514-519.

[34] Ziakas NG, Boboridis K, Nakos E, Mikropoulos D, Margaritis V, et al (2009) Does the use of trypan blue during phacoemulsification affect the intraocular pressure? Can. J. Ophthalmol. 44:293-296.

[35] Yamakiri K, Sakamoto T, Noda Y, Nakahara M, Ogino N, et al (2007) Reduced incidence of intraoperative complications in a multicenter controlled clinical trial of triamcinolone in vitrectomy. Ophthalmology. 114:289-296. 
[36] Moshfeghi DM, Kaiser PK, Scott IU, Sears JE, Benz M, et al (2003) Acute endophthalmitis following intravitreal triamcinolone acetonide injection. Am. J. Ophthalmol. 136:791-796.

[37] Chang YS, Tseng SY, Tseng SH, Wu CL, Chen MF (2006) Triamcinolone acetonide suspension toxicity to corneal endothelial cells. J. Cataract Refract. Surg. 32:1549-1555.

[38] Oh Y, Wee WR, Lee JH, Kim MK (2007) Short-term effect of intracameral triamcinolone acetonide on corneal endothelium using the rabbit model. Eye 21.812-818. 